\title{
Protective role of lidocaine against cerebral ischemia-reperfusion injury: An in vitro study
}

\author{
XIAOYANG LAN ${ }^{1}$ and YUMIN XU ${ }^{2}$ \\ ${ }^{1}$ Department of Neurology, First Medical Center, People's Liberation Army General Hospital, Beijing 100853; \\ ${ }^{2}$ Department of Anesthesiology, Taizhou People's Hospital, Taizhou, Jiangsu 225300, P.R. China
}

Received November 19, 2019; Accepted May 20, 2020

DOI: 10.3892/etm.2021.10964

\begin{abstract}
Lidocaine, a local anesthetic, is a valuable agent for the treatment of neuronal ischemia/reperfusion (I/R) injury. The aim of the present study was to investigate the role of lidocaine in oxygen-glucose deprivation/reperfusion (OGD/R)-induced cortical neurons and explore the related molecular mechanisms. Cerebral cortical neurons were isolated from Sprague-Dawley rat embryos and stimulated with $\mathrm{OGD} / \mathrm{R}$ to establish an in vitro I/R injury model. Subsequently, neuronal cell viability, cytotoxicity and apoptosis were evaluated by performing the MTT assay, lactate dehydrogenase (LDH) assay and flow cytometry, respectively. The results suggested that OGD/R exposure significantly decreased cerebral cortical neuron cell viability, accelerated LDH release and induced cell apoptosis compared with control neurons, indicating that cerebral I/R injury was stimulated by OGD/R treatment. Further investigation indicated that $10 \mu \mathrm{M}$ lidocaine significantly enhanced neuronal cell viability, and reduced LDH release and neuronal cell apoptosis in OGD/R-exposed cells compared with the OGD/R + saline group, which indicated that lidocaine displayed neuroprotective effects against I/R damage. In addition, the findings of the present study suggested that OGD/R exposure significantly decreased Bcl-2 and Bcl-xl protein expression levels, but increased Bax protein expression levels, the Bax/Bcl-2 ratio and caspase-3 activity compared with control neurons. However, lidocaine reversed OGD/R-mediated alterations to apoptosis-related protein expression. Furthermore, the results of the present study indicated that lidocaine increased Wnt3a, $\beta$-catenin and cyclin D1 expression levels in OGD/R-exposed cells compared with the OGD/R + saline group, thus activating the $\mathrm{Wnt} / \beta$-catenin signaling pathway. The findings of the present study suggested that lidocaine served a protective role in OGD/R-triggered neuronal damage by activating the
\end{abstract}

Correspondence to: Mr. Yumin Xu, Department of Anesthesiology, Taizhou People's Hospital, 210 Yingchun Road, Taizhou, Jiangsu 225300, P.R. China

E-mail: xuyumin19112@163.com

Key words: lidocaine, cerebral ischemia-reperfusion injury, cerebral cortical neurons
Wnt/ $\beta$-catenin signaling pathway; therefore, lidocaine may serve as a potential candidate for the treatment of cerebral I/R injury.

\section{Introduction}

Ischemia/reperfusion (I/R) injury is caused by deficient oxygen supply followed by restoration of blood circulation. $\mathrm{I} / \mathrm{R}$ is the primary cause of cerebral damage and is a major clinical problem in cerebral injury therapy $(1,2)$. Previous studies have demonstrated that deficient oxygen and glucose supply may result in neuronal injury during ischemic brain damage $(3,4)$. Previous studies have also demonstrated that oxygen-glucose deprivation (OGD) has detrimental effects on the cells, including oxidative stress and immoderate glutamate release, resulting in toxic levels $(5,6)$. However, the specific pathogenesis of cerebral I/R injury and the possible signaling pathways participating in ischemic neuronal injury are not completely understood.

A number of studies have indicated that numerous anesthetic drugs exert neuroprotective effects in cerebral I/R injury, including isoflurane (7), propofol (8) and curcumin (9). Lidocaine, a widely used local anesthetic, may improve the cognitive ability of patients suffering from cardiopulmonary conditions (10). In addition, the protective effects of lidocaine on ischemia- or hypoxia-stimulated neuronal injury are mediated by diverse mechanisms, including vasodilation, improvement of the microcirculation and suppression of platelet aggregation $(11,12)$. Although the neuroprotective role of lidocaine in brain $\mathrm{I} / \mathrm{R}$ damage has been previously demonstrated in in vitro and in vivo models $(13,14)$, the mechanisms underlying the neuroprotective properties of lidocaine remain to be elucidated. Therefore, the aim of the present study was to investigate the functions of lidocaine in an in vitro $\mathrm{I} / \mathrm{R}$ model.

Various signaling pathways have been associated with the pathogenesis of cerebral I/R, including mitogen-activated protein kinase (15), N-methyl D-aspartate receptor subtype 2B/ERK/cAMP response element-binding protein (16), Janus kinase 2/STAT3 (17) and Wnt/ $\beta$-catenin (18) signaling pathways. The Wnt signaling pathway is the most common signaling pathway that regulates and mediates a series of cellular processes, including proliferation and apoptosis (19). The canonical Wnt/ $\beta$-catenin signaling pathway serves a 
key role in cell survival following cerebral ischemia (18). Previous studies have demonstrated that activation of the Wnt/ $\beta$-catenin signaling pathway during I/R exerts corresponding organ-protective effects (20-22). However, whether lidocaine affects the $\mathrm{Wnt} / \beta$-catenin signaling pathway in $\mathrm{I} / \mathrm{R}$ injury is not completely understood.

The aim of the present study was to investigate the roles and mechanisms underlying the action of lidocaine in OGD/reperfusion (OGD/R)-induced cortical neurons.

\section{Materials and methods}

Primary culture of cortical neurons. Brain cortical neurons were obtained from Sprague-Dawley rats (age, embryonic day 18; SD rats were obtained from the Model Animal Research Center of Nanjing University) according to previous study (23). In brief, cerebral cortices were separated and digested with trypsin (Gibco; Thermo Fisher Scientific, Inc.) at $37^{\circ} \mathrm{C}$ for $15 \mathrm{~min}$, re-tempered in DMEM (Gibco; Thermo Fisher Scientific, Inc.) supplemented with 10\% FBS (Gibco; Thermo Fisher Scientific, Inc.) and 1\% penicillin/streptomycin (Gibco; Thermo Fisher Scientific, Inc.) and filtered through a 40-mm nylon mesh. Cells were cultured in a 6-well poly-L-lysine coated culture plate (Sigma-Aldrich; Merck KGaA) and maintained at $37^{\circ} \mathrm{C}$ in an incubator in DMEM containing $2 \%$ B27 (Gibco; Thermo Fisher Scientific, Inc.), $0.5 \mathrm{mM}$ glutamine and $100 \mathrm{U} / \mathrm{ml}$ penicillin/streptomycin. Following culture at $37^{\circ} \mathrm{C}$ for $24 \mathrm{~h}, 10 \mathrm{mM}$ cytosine arabinoside (Sigma-Aldrich; Merck $\mathrm{KGaA}$ ) was applied to inhibit non-neuronal cell survival and neurons were cultured at $37^{\circ} \mathrm{C}$ for an additional 2 days. The mother rats $(n=3$; Model Animal Research Center of Nanjing University, Nanjing, China) were anaesthetized with $2 \%$ halothane prior to sacrifice by cervical dislocation. The depth of anesthesia was monitored by the toe pinch method. Death was verified by monitoring cessation of the heartbeat and breathing. The fetuses $(n=6)$ were also anaesthetized with $2 \%$ halothane prior to sacrifice by cervical dislocation. All rats were housed under standard conditions at room temperature $\left(22-24^{\circ} \mathrm{C}\right)$ and $60-65 \%$ humidity, on a 12 -h light/dark cycle with ad libitum supply of food and water. The present study was approved by the Animal Ethics Committee of the First Medical Center, People's Liberation Army General Hospital. All experiments conformed to the guidelines for the Care and Use of Laboratory Animals of the National Institutes of Health (24).

Neuronal I/R injury model establishment and lidocaine stimulation. The OGD/R model was constructed as previously described (25). To induce OGD, brain cortical neurons were cultured in Earle's Balanced Salt Solution (Thermo Fisher Scientific, Inc.) and transferred into a hypoxia chamber for 4 h with $95 \% \mathrm{~N}_{2}$ and $5 \% \mathrm{CO}_{2}$ at $37^{\circ} \mathrm{C}$. Subsequently, cells were cultured in an environment of $5 \% \mathrm{CO}_{2}$ and $95 \%$ air at $37^{\circ} \mathrm{C}$ for $24 \mathrm{~h}$. To induce $\mathrm{R}$, following culture in the hypoxic chamber, cells were cultured for $24 \mathrm{~h}$ in HyClone medium 199 (Cytiva) supplemented with $2 \% \mathrm{~B} 27$ and $0.5 \mathrm{mM}$ glutamine in normoxic conditions $\left(95 \%\right.$ air $\left./ 5 \% \mathrm{CO}_{2}\right)$ at $37^{\circ} \mathrm{C}$. For lidocaine stimulation, cultured cortical neurons were treated with $10 \mu \mathrm{M}$ lidocaine, as previously described (26), or saline at $37^{\circ} \mathrm{C}$ for $4 \mathrm{~h}$ after exposure to OGD/R injury.
MTT assay. Following treatment, MTT solution was added to each well $\left(5 \times 10^{4}\right.$ cells per well) and incubated for $4 \mathrm{~h}$ at $37^{\circ} \mathrm{C}$. The supernatant was discarded and $100 \mu 1$ DMSO was added to dissolve blue formazan crystals. The absorbance of each well was measured at a wavelength of $570 \mathrm{~nm}$ using a microplate reader (Jupiter G19060; Montréal Biotech, Inc.)

Lactate dehydrogenase ( $\mathrm{LDH}$ ) analysis. $\mathrm{LDH}$ production in cells was determined using an LDH-Cytotoxicity assay kit (Sigma-Aldrich; Merck KGaA). Briefly, the supernatant of cerebral cortical neurons was collected from each well after treatment through centrifugation $\left(400 \mathrm{x} \mathrm{g}, 5 \mathrm{~min}, 4^{\circ} \mathrm{C}\right)$ and subjected to the assay according to the manufacturer's protocol at room temperature for $15 \mathrm{~min}$. The absorbance of each sample was measured at a wavelength of $490 \mathrm{~nm}$ using the FLUOstar ${ }^{\circledR}$ Omega Microplate Reader (BMG Labtech $\mathrm{GmbH}$ ).

Flow cytometry analysis. Following OGD/R and lidocaine or saline treatment, the cerebral cortical neurons were collected for Annexin V-FITC/PI (BD Biosciences) double staining, according to the manufacturer's instructions. Neuronal cell apoptosis (early + late apoptosis) was evaluated by flow cytometry using a BD FACSCalibur flow cytometer (BD Biosciences) and FlowJo software (version 7.2.4; FlowJo LLC).

Determination of caspase-3 activity. To determine caspase 3 activity, a Caspase-3 Assay kit (cat. no. ab39401; Abcam) was used according to the manufacturer's protocol. Briefly, cells were resuspended in $50 \mu \mathrm{l}$ of chilled Cell Lysis Buffer (Abcam) and incubated the cells on ice for $10 \mathrm{~min}$. Then cells were centrifuged at 10,000 x g for $1 \mathrm{~min}$. Subsequently, supernatant (cytosolic extract) was transferred to a fresh tube and put on ice. Subsequently, an automatic micro-plate reader (ELX800; BioTek Instruments, Inc.) was used to spectrophotometrically determine caspase-3 activity levels at a wavelength of $405 \mathrm{~nm}$ according to the manufacturer's protocol.

Reverse transcription-quantitative PCR (RT-qPCR) analysis. Following the indicated treatment, total RNA was extracted from cerebral cortical neurons $\left(10^{7}\right.$ cells per well $)$ using TRIzol ${ }^{\circledR}$ reagent (Invitrogen; Thermo Fisher Scientific, Inc.) according to the manufacturer's protocol. Total RNA was reverse transcribed into cDNA using the PrimeScript ${ }^{\mathrm{TM}}$ RT reagent kit (Takara Bio, Inc.). The temperature protocol for reverse transcription was as follows: $70^{\circ} \mathrm{C}$ for $5 \mathrm{~min}, 37^{\circ} \mathrm{C}$ for $5 \mathrm{~min}$ and $42^{\circ} \mathrm{C}$ for $1 \mathrm{~h}$. Subsequently, qPCR was performed using an ABI 7000 Real-Time PCR system (Applied Biosystems; Thermo Fisher Scientific, Inc.) with SYBR-Green PCR Master Mix Kit (Takara Bio, Inc.). The thermocycling conditions used for qPCR were as follows: Initial denaturation at $95^{\circ} \mathrm{C}$ for $5 \mathrm{~min}$; 40 cycles at $95^{\circ} \mathrm{C}$ for $15 \mathrm{sec}$ and $60^{\circ} \mathrm{C}$ for $60 \mathrm{sec}$; and a final extension at $72^{\circ} \mathrm{C}$ for $30 \mathrm{sec}$. The following primers were used for qPCR (Sangon Biotech Co., Ltd.): Wnt3a forward, 5'-AACTGCACCACCGTCCAC-3' and reverse, 5'-AAGGCC GACTCCCTGGTA-3'; $\beta$-catenin forward, 5'-AACAGGGTC TGGGACATTAGTC-3' and reverse, 5'-CGAAAGCCAATC AAACACAAAC-3'; cyclin D1 forward, 5'-AACTACCTG GACCGCTTCCT-3' and reverse, 5'-CCACTTGAGCTTGTT CACCA-3'; and GAPDH forward, 5'-TGTTGCCATCAATGA CCCCTT-3' and reverse, 5'-CTCCACGACGTACTCAGCG-3'. 

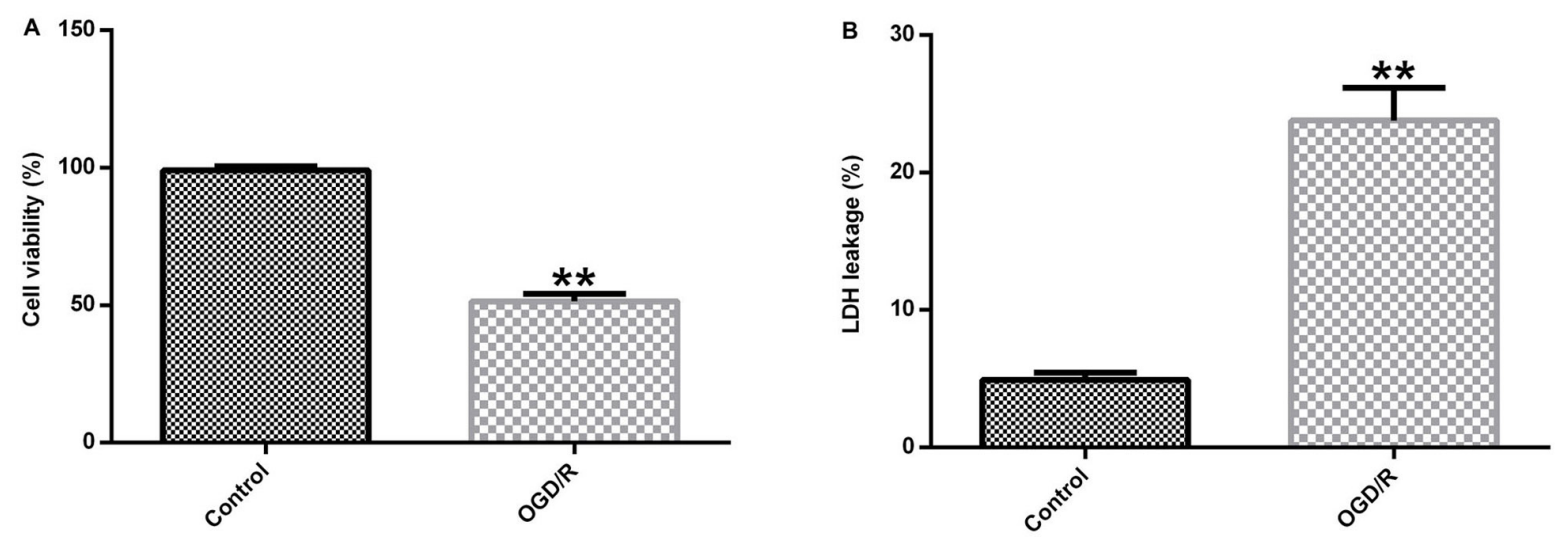

C
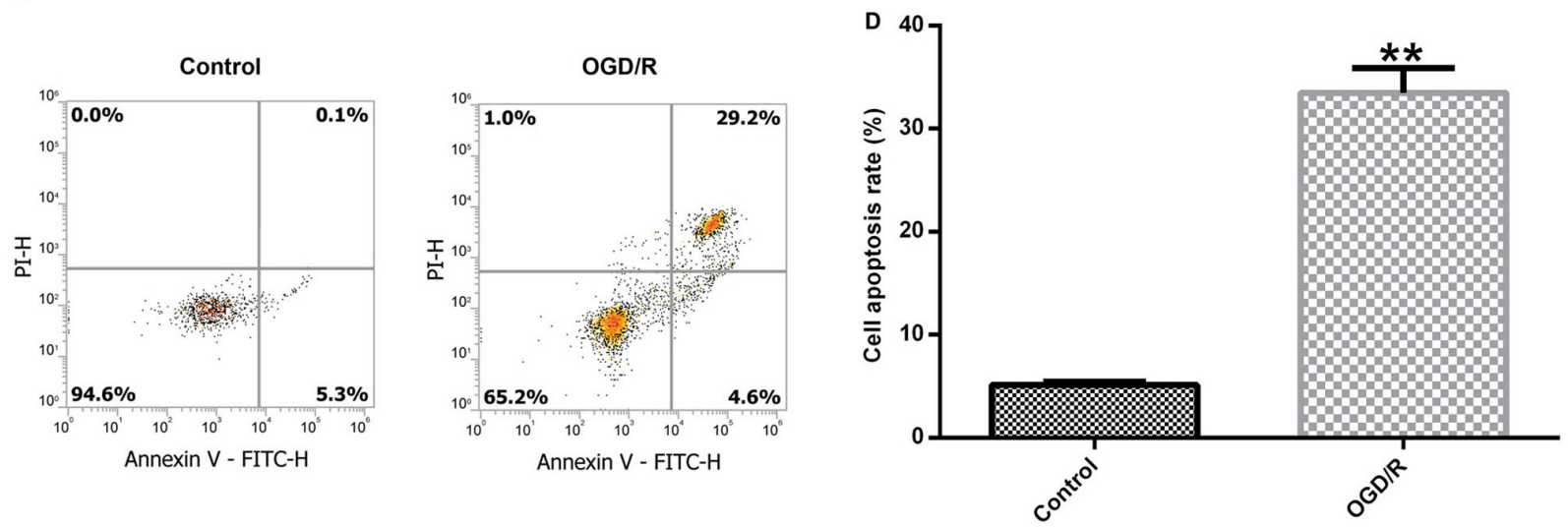

Figure 1. Establishment of the in vitro neuronal cerebral I/R injury model. Primary cerebral cortical neurons were isolated from Sprague-Dawley rat embryos and OGD/R was used to construct a cerebral I/R injury model in vitro. (A) Cell viability was evaluated by performing the MTT assay. (B) Cell injury was assessed by measuring LDH release. The proportion of apoptotic cells was (C) determined by flow cytometry and (D) quantified. ${ }^{* *} \mathrm{P}<0.01 \mathrm{vs.} \mathrm{control.}$ $\mathrm{I} / \mathrm{R}$, ischemia-reperfusion; OGD/R, oxygen-glucose deprivation/reoxygenation; LDH, lactate dehydrogenase; PI, propidium iodide.

mRNA expression levels were quantified using the $2^{-\Delta \Delta \mathrm{Cq}}$ method (27) and normalized to the internal reference gene GAPDH.

Western blotting. The cerebral cortical neurons were treated as indicated. Total protein was extracted from cerebral cortical neurons using RIPA lysis buffer (Sigma-Aldrich; Merck $\mathrm{KGaA}$ ). Total protein was quantified using the BCA Protein assay kit (Sigma-Aldrich; Merck KGaA). Proteins ( $40 \mu \mathrm{g} / \mathrm{lane})$ were subjected to $10 \%$ SDS-PAGE and transferred onto PVDF membranes (EMD Millipore). The membranes were blocked in 5\% fat-free milk at room temperature for $1.5 \mathrm{~h}$. Subsequently, the membranes were incubated overnight at $4^{\circ} \mathrm{C}$ with primary antibodies targeted against: Wnt3a (cat. no. ab219412; 1:1,000; Abcam), $\beta$-catenin (cat. no. ab32572; 1:1,000; Abcam), cyclin D1 (cat. no. ab16663; 1:1,000; Abcam), Bcl-2 (cat. no. ab196495; 1:1,000; Abcam), Bax (cat. no. ab32503; 1:1,000; Abcam), Bcl-xl (cat. no. ab32370; 1:1,000; Abcam) and GAPDH (cat. no. ab181602; 1:1,000; Abcam). Subsequently, the membranes were incubated with a horseradish peroxidase-conjugated secondary antibody (cat. no. ab7090; 1:2,000; Abcam) at room temperature for $2 \mathrm{~h}$. Finally, the protein bands were observed by chemiluminescence using the ECL Advance Western Blotting Detection kit (Cytiva). Protein expression levels were quantified using ImageJ software (version 1.52s; National Institutes of Health).
Statistical analysis. SPSS (version 21.0; IBM Corp.) was used to perform statistical analyses. All data are presented as the mean \pm standard deviation from three independent experiments. Comparisons between two groups were analyzed using the unpaired Student's t-test. Comparisons among multiple groups were analyzed using one-way ANOVA followed by Tukey's post hoc test. $\mathrm{P}<0.05$ was considered to indicate a statistically significant difference.

\section{Results}

$O G D / R$ successfully induces a cerebral I/R injury model. Brain cortical neurons were cultured under OGD conditions for $4 \mathrm{~h}$ and exposed to $\mathrm{R}$ conditions for $24 \mathrm{~h}$ to induce cerebral I/R injury in vitro. Subsequently, cell viability, LDH release and cell apoptosis were detected to assess neuronal injury. Cerebral cortical neuronal cell viability was significantly decreased in the OGD/R group compared with the control group (Fig. 1A). In addition, OGD/R-exposed cortical neurons displayed significantly increased LDH release compared with control cortical neurons (Fig. 1B). Furthermore, the level of apoptosis in control and OGD/R-stimulated cells was determined. OGD/R treatment notably enhanced cell apoptosis and significantly increased the percentage of apoptotic neurons in the OGD/R group compared with the control group (Fig. 1C and D). In summary, the results demonstrated that OGD/R successfully induced the neuronal $\mathrm{I} / \mathrm{R}$ injury model in vitro. 

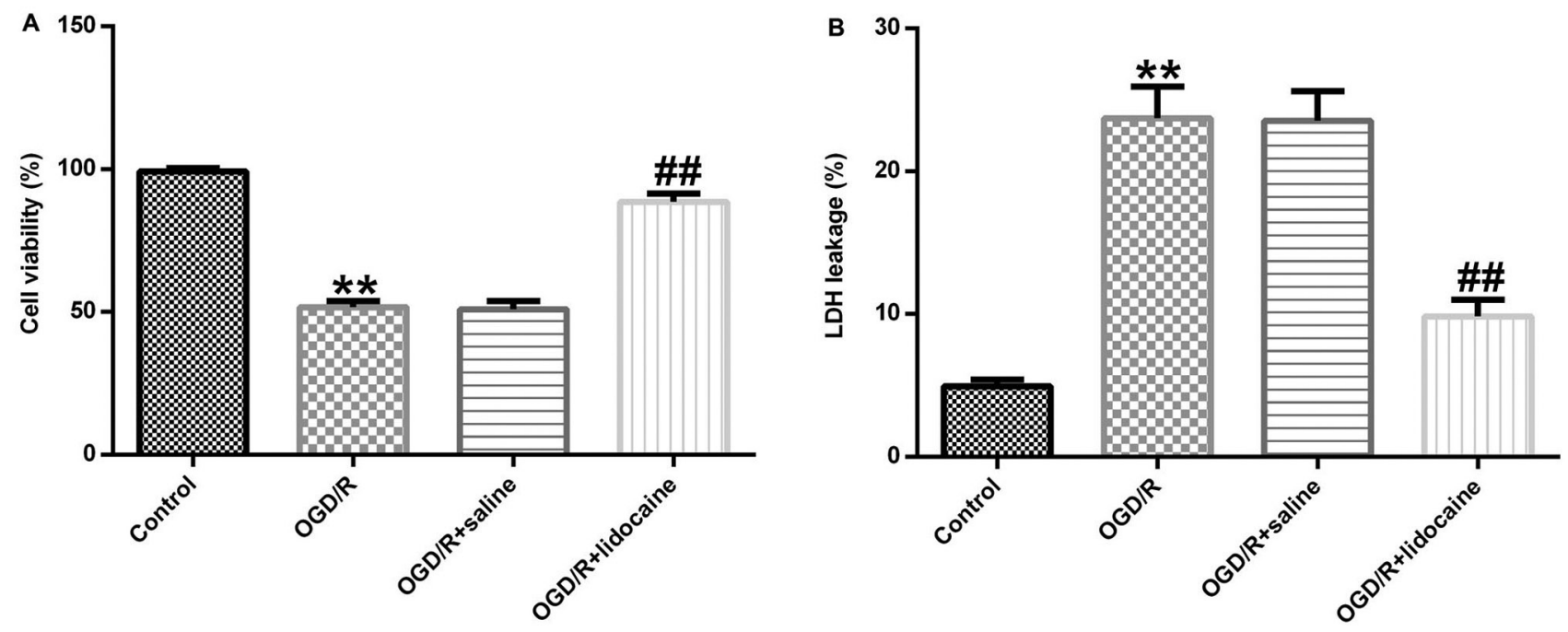

Figure 2. Effects of lidocaine on the viability and LDH release of OGD/R-induced neurons. OGD/R-exposed cerebral cortical neurons were treated with $10 \mu \mathrm{g} / \mathrm{ml}$ lidocaine or saline. Cells were divided into four groups: i) Control; ii) OGD/R; iii) OGD/R + saline; and iv) OGD/R + lidocaine. (A) Cell viability was evaluated by performing the MTT assay. (B) Cell injury was assessed by measuring $\mathrm{LDH}$ release. ${ }^{* *} \mathrm{P}<0.01$ vs. control; ${ }^{\# \#} \mathrm{P}<0.01 \mathrm{vs}$. OGD/R + saline. $\mathrm{LDH}$, lactate dehydrogenase; OGD/R, oxygen-glucose deprivation/reoxygenation.

Lidocaine exerts neuroprotective effects against $O G D / R$-induced neuronal injury. The role of lidocaine in OGD/R-stimulated neuronal damage was investigated. Cerebral cortical neurons were treated with $10 \mu \mathrm{M}$ lidocaine or saline for $4 \mathrm{~h}$ following exposure to OGD/R. Cell viability was evaluated by performing the MTT assay. Cell viability in the OGD/R group was significantly decreased compared with the control group, whereas cell viability was significantly enhanced in the $\mathrm{OGD} / \mathrm{R}+$ lidocaine group compared with the OGD/R + saline group (Fig. 2A). In addition, LDH release was measured to assess cell toxicity. The results indicated that $\mathrm{OGD} / \mathrm{R}$ exposure significantly increased LDH release from cerebral cortical neurons compared with the control group, whereas lidocaine reversed this effect (Fig. 2B). The results demonstrated that lidocaine increased cell viability and reduced LDH secretion in the OGD/R-induced neuronal injury model, indicating that lidocaine suppressed neuronal injury.

Lidocaine inhibits $O G D / R$-induced neuronal cell apoptosis. To further elucidate the mechanism underlying the effects of lidocaine in OGD/R-triggered neuronal injury, neuronal cells were subjected to OGD/R and then treated with $10 \mu \mathrm{M}$ lidocaine or saline. Neuronal cell apoptosis was assessed by flow cytometry. The number of apoptotic neurons was notably higher in the OGD/R group compared with the control group (Fig. 3A). However, the number of apoptotic neurons was obviously reduced in the $\mathrm{OGD} / \mathrm{R}+$ lidocaine group compared with the OGD/R + saline group (Fig. 3A). The percentage of apoptotic neurons was also quantified in the different groups. The number of apoptotic neurons was significantly higher in the OGD/R group compared with the control group, but significantly lower in the $\mathrm{ODG} / \mathrm{R}+$ lidocaine group compared with the ODG/R + saline group (Fig. 3B). Neuronal apoptosis is regulated by apoptosis-specific proteins, including Bcl-2, Bax and Bcl-xl (28); thus, the expression of apoptosis-related proteins in the different groups was detected by western blotting. The western blotting results indicated that $\mathrm{OGD} / \mathrm{R}$ markedly decreased Bcl-2 and Bcl-xl protein expression levels, but increased Bax protein expression levels compared with the control group. However, lidocaine reversed OGD/R-mediated apoptosis-related protein expression (Fig. 3C). The Bax/Bcl-2 ratio was also significantly increased by $\mathrm{OGD} / \mathrm{R}$ treatment compared with the control group, whereas lidocaine treatment reduced the effect of OGD/R on the Bax/Bcl-2 ratio (Fig. 3D). $\mathrm{OGD} / \mathrm{R}$-mediated reductions in $\mathrm{Bcl}-\mathrm{xl}$ protein expression were inhibited by lidocaine treatment (Fig. 3E). Compared with the control group, caspase-3 activity was also significantly increased by $\mathrm{OGD} / \mathrm{R}$ treatment, but lidocaine treatment reversed OGD/R-mediated alterations to caspase-3 activity (Fig. 3F). The results indicated the potential role of lidocaine in $\mathrm{OGD} / \mathrm{R}$-mediated neuronal injury.

Lidocaine regulates the activation of the Wnt/ $\beta$-catenin signaling pathway in $O G D / R$-induced neurons. Finally, the potential mechanism underlying the effects of lidocaine on OGD/R-triggered neurons was investigated. The western blotting results indicated that Wnt $3 \mathrm{a}, \beta$-catenin and cyclin D1 protein expression levels were significantly reduced in OGD/R-stimulated neurons compared with control neurons. However, lidocaine significantly upregulated the expression of the three proteins in OGD/R-exposed neurons compared with the OGD/R + saline group (Fig. 4A-D). Similar results were obtained for mRNA expression levels of Wnt3a, $\beta$-catenin and cyclin D1 (Fig. 4E-G). Collectively, the results suggested that Wnt//-catenin signaling may be involved in OGD/R-mediated neuron injury, and lidocaine displayed neuroprotective activity in OGD/R-exposed neurons, at least partly by activating the Wnt/ $\beta$-catenin signaling pathway.

\section{Discussion}

$\mathrm{I} / \mathrm{R}$ injury is a major health concern in the clinic (29); therefore, designing an effective treatment strategy for cerebral I/R injury is of clinical significance. According to previous studies, deficient oxygen and glucose supply may contribute to neuronal 

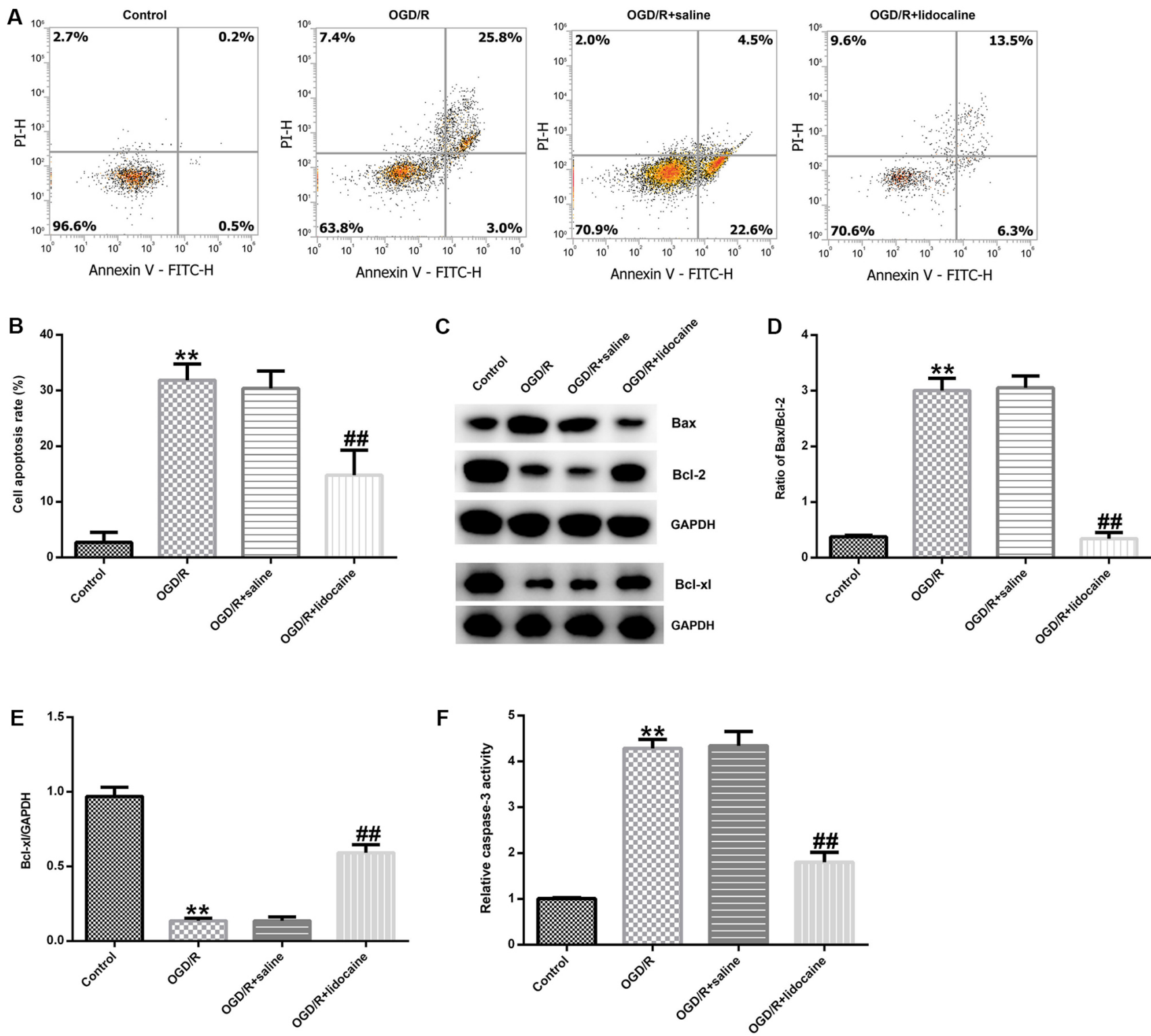

Figure 3. Effects of lidocaine on OGD/R-induced neuronal cell apoptosis. Cerebral cortical neurons were subjected to OGD/R and then treated with $10 \mu \mathrm{g} / \mathrm{ml}$ lidocaine or saline. Cells were divided into four groups: i) Control; ii) OGD/R; iii) OGD/R + saline; and iv) OGD/R + lidocaine. The proportion of apoptotic neurons was (A) determined by flow cytometry and (B) quantified. Protein expression were (C) determined by western blotting and semi-quantified for (D) the ratio of Bax/Bcl-2 and (E) Bcl-xl. (F) The relative caspase-3 activity. ${ }^{* *} \mathrm{P}<0.01$ vs. control; ${ }^{\# \#} \mathrm{P}<0.01 \mathrm{vs}$. OGD/R + saline. OGD/R, oxygen-glucose deprivation/reoxygenation; PI, propidium iodide.

injury during ischemic brain damage $(3,4,30)$. OGD may have detrimental effects on cell function, such as oxidative stress and immoderate glutamate release, resulting in toxic levels (31). In previous studies, cerebral cortical neurons were stimulated with $\mathrm{OGD} / \mathrm{R}$ to induce an in vitro cerebral I/R injury model $(32,33)$. In the present study, cortical neurons were isolated from Sprague-Dawley rat embryos and OGD/R was used to establish an in vitro I/R injury model. Subsequently, cell viability, LDH release and cell apoptosis were detected to evaluate neuronal cell damage. The results indicated that $\mathrm{OGD} / \mathrm{R}$ exposure significantly decreased cerebral cortical neuronal viability, increased LDH release and induced cell apoptosis compared with control neurons. The results also indicated that OGD/R successfully established an in vitro I/R injury model. However, the staining of NSE of primary cerebral cortical neurons was not conducted in the present study, which was a key limitation.
Previous studies have demonstrated that various elements serve neuroprotective roles in cerebral I/R injury models, such as tamibarotene (34), daucosterol (35) and orally administered crocin (36). Zhang et al (36) reported that orally administered crocin exerted protective effects against cerebral I/R injury via metabolic transformation of crocetin by the gut microbiota. Tian et al (34) observed that tamibarotene improves hippocampal injury stimulated by focal cerebral I/R via the PI3K/Akt signaling pathway in rats. Lidocaine, a local anesthetic that is widely used in surgery, is frequently used to treat neurovascular diseases and serves pivotal roles in the prevention and treatment of ischemic brain injury (37). However, the neuroprotective effects of lidocaine and the underlying mechanisms in OGD/R-stimulated injury are not completely understood. Therefore, the present study investigated the neuroprotective mechanisms underlying the effects of lidocaine in cerebral I/R neurons in vitro. 

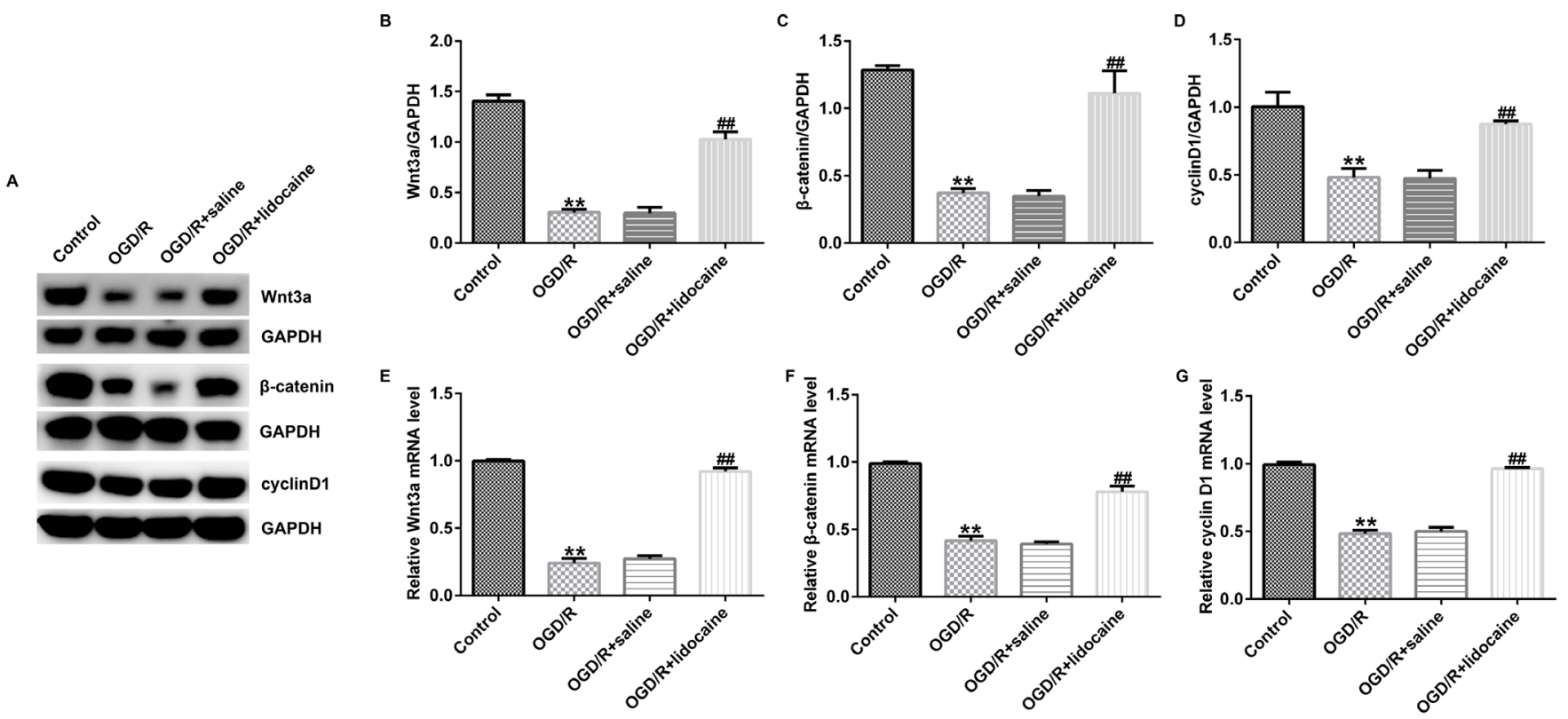

Figure 4. Effects of lidocaine on OGD/R-mediated regulation of the Wnt/ $\beta$-catenin signaling pathway. OGD/R-exposed cerebral cortical neurons were treated with $10 \mu \mathrm{g} / \mathrm{ml}$ lidocaine or saline. Protein expression levels were (A) determined by western blotting and semi-quantified for (B) Wnt3a, (C) $\beta$-catenin and (D) cyclin D1. The mRNA expression levels of (E) Wnt3a, (F) $\beta$-catenin and (G) cyclin D1 were evaluated by reverse transcription-quantitative PCR. ${ }^{* *} \mathrm{P}<0.01$ vs. control; ${ }^{\# \#} \mathrm{P}<0.01$ vs. OGD/R + saline. OGD/R, oxygen-glucose deprivation/reoxygenation.

To evaluate the role of lidocaine in OGD/R-induced neuronal cell injury, MTT and LDH release assays were performed. The results demonstrated that $10 \mu \mathrm{M}$ lidocaine significantly enhanced cell viability and reduced LDH release in OGD/R-induced cerebral cortical neurons compared with the OGD/R + saline group. Therefore, the results indicated that lidocaine relieved OGD/R-induced neuronal cell injury. It was previously suggested that lidocaine may be beneficial to the nervous system by regulating cell apoptosis (38). In the present study, flow cytometry was conducted to evaluate apoptosis in the OGD/R-induced neuronal cell injury model and the results indicated that the number of apoptotic neurons was significantly decreased in OGD/R-induced neurons following lidocaine treatment compared with saline treatment. Neuronal cell apoptosis is usually triggered by interactions between Bcl-2 family members via endogenic apoptotic cascades $(39,40)$. In addition, the $\mathrm{Bax} / \mathrm{Bcl}-2$ ratio is considered a key indicator of apoptotic stimulation (41). In the present study, lidocaine enhanced Bcl-2 and Bcl-xl protein expression levels, but decreased Bax expression levels, the Bax/Bcl-2 ratio and caspase-3 activity in OGD/R-stimulated neurons compared with the OGD/R + saline group. Multiple signaling pathways are associated with the pathogenesis of cerebral I/R, including the Wnt/ $\beta$-catenin signaling pathway $(18,42)$. To further investigate the mechanism underlying the neuroprotective effects of lidocaine, RT-qPCR and western blotting were conducted to evaluate the relative mRNA and protein levels of Wnt/ $\beta$-catenin signaling pathway components. The results demonstrated that the mRNA and protein expression levels of Wnt3a, $\beta$-catenin and cyclin D1 were significantly increased in OGD/R-exposed neurons treated with $10 \mu \mathrm{M}$ lidocaine compared with OGD/R-exposed neurons treated with saline; however, immunofluorescence staining was not performed to verify the results, which may be a limitation of the present study. Taken together, the results indicated that lidocaine treatment promoted neuronal viability, and reduced LDH release and neuronal cell apoptosis by regulating the Wnt/ $\beta$-catenin signaling pathway. However, the effects of lidocaine on I/R injury were not studied in vivo in the present study, which was a further a limitation of the present study and requires further investigation.

The findings of the present study may provide novel insight into the mechanism underlying the neuroprotective effects of lidocaine against OGD/R-induced neuronal damage via activating the $\mathrm{Wnt} / \beta$-catenin signaling pathway. However, the present study was only a preliminary in vitro study of the effects of lidocaine on I/R injury. In order to verify the results of the present study, further in-depth research is required. For example, an inhibitor of $\mathrm{Wnt} / \beta$-catenin signaling pathway should be applied to investigate whether lidocaine directly regulates the Wnt/ $\beta$-catenin signaling pathway. Furthermore, only one dose of lidocaine $(10 \mu \mathrm{g} / \mathrm{ml})$ was used in the present study to investigate the effect of lidocaine on I/R injury in vitro, which is a further limitation of the present study. Therefore, future studies should investigate the effect of different doses of lidocaine on $\mathrm{I} / \mathrm{R}$ injury. In addition, the effect of lidocaine on I/R injury should also be investigated in vivo.

In conclusion, the present study indicated that lidocaine suppressed OGD/R-induced neuronal cell injury and apoptosis by stimulating the $\mathrm{Wnt} / \beta$-catenin signaling pathway, thereby exerting a protective effect against OGD/R-induced neuronal injury. Therefore, lidocaine may serve as a potential therapeutic candidate for I/R injury.

\section{Acknowledgements}

Not applicable. 


\section{Funding}

No funding was received.

\section{Availability of data and materials}

The datasets used and/or analyzed during the current study are available from the corresponding author on reasonable request.

\section{Authors' contributions}

XL contributed to study design, data collection, statistical analysis, data interpretation and manuscript preparation. YX contributed to data collection and statistical analysis and manuscript preparation. XL and YX confirmed the authenticity of all the raw data. All authors have read and approved the final manuscript.

\section{Ethics approval and consent to participate}

The present study was approved by the Animal Ethics Committee of the First Medical Center, People's Liberation Army General Hospital. All experiments conformed to the guidelines for the Care and Use of Laboratory Animals of the National Institutes of Health.

\section{Patient consent for publication}

Not applicable.

\section{Competing interests}

The authors declare that they have no competing interests.

\section{References}

1. Papadakis M and Buchan A: Approaches to neuroprotective and reperfusion injury therapy. Handb Clin Neurol 94: 1205-1223, 2009.

2. Zhào H,Liu Y,Zeng J,Li D,Zhang W and Huang Y: Troxerutin and cerebroprotein hydrolysate injection protects neurovascular units from oxygen-glucose deprivation and reoxygenation-induced injury in vitro. Evid Based Complement Alternat Med 2018: 9859672, 2018

3. Zhuonan Z, Sen G, Zhipeng J, Maoyou Z, Linglan Y, Gangping W, Cheng J, Zhongliang M, Tian J, Peijian Z and Kesen X: Hypoxia preconditioning induced HIF-1alpha promotes glucose metabolism and protects mitochondria in liver I/R injury. Clin Res Hepatol Gastroenterol 39: 610-619, 2015.

4. Yin $X$, Feng L, Ma D, Yin P, Wang X, Hou S, Hao Y, Zhang J, Xin M and Feng J: Roles of astrocytic connexin-43, hemichannels, and gap junctions in oxygen-glucose deprivation/reperfusion injury induced neuroinflammation and the possible regulatory mechanisms of salvianolic acid B and carbenoxolone. J Neuroinflammation 15: 97, 2018.

5. Park SJ, Jung YJ, Kim YA, Lee-Kang JH and Lee KE: Glucose/oxygen deprivation and reperfusion upregulate SNAREs and complexin in organotypic hippocampal slice cultures. Neuropathology 28: 612-620, 2008.

6. Jung YJ, Park SJ, Park JS and Lee KE: Glucose/oxygen deprivation induces the alteration of synapsin I and phosphosynapsin. Brain Res 996: 47-54, 2004.

7. Wang S, Yin J, Ge M, Dai Z, Li Y, Si J, Ma K, Li L and Yao S: Transforming growth-beta 1 contributes to isoflurane postconditioning against cerebral ischemia-reperfusion injury by regulating the c-Jun N-terminal kinase signaling pathway. Biomed Pharmacother 78: 280-290, 2016.
8. Yu W, Gao D, Jin W, Liu S and Qi S: Propofol prevents oxidative stress by decreasing the ischemic accumulation of succinate in focal cerebral ischemia-reperfusion injury. Neurochem Res 43: 420-429, 2018

9. Hou Y, Wang J and Feng J: The neuroprotective effects of curcumin are associated with the regulation of the reciprocal function between autophagy and HIF-1alpha in cerebral ischemia-reperfusion injury. Drug Des Devel Ther 13: 1135-1144, 2019.

10. Zeng ZW, Zhang YN, Lin WX, Zhang WQ and Luo R: A meta-analysis of pharmacological neuroprotection in noncardiac surgery: Focus on statins, lidocaine, ketamine, and magnesium sulfate. Eur Rev Med Pharmacol Sci 22: 1798-1811, 2018.

11. Wen X, Xu S, Zhang Q, Li X, Liang H, Yang C, Wang H and Liu H: Inhibitory gene expression of the Cav3.1 T-type calcium channel to improve neuronal injury induced by lidocaine hydrochloride. Eur J Pharmacol 775: 43-49, 2016.

12. Mitchell SJ and Merry AF: Lignocaine: Neuro-protective or wishful thinking? J Extra Corpor Technol 41: P37-P42, 2009.

13. Naito H, Takeda Y, Danura T, Kass IS and Morita K: Effect of lidocaine on dynamic changes in cortical reduced nicotinamide adenine dinucleotide fluorescence during transient focal cerebral ischemia in rats. Neuroscience 235: 59-69, 2013.

14. Zhang Y and Lipton P: Cytosolic $\mathrm{Ca}^{2+}$ changes during in vitro ischemia in rat hippocampal slices: Major roles for glutamate and $\mathrm{Na}-$-dependent $\mathrm{Ca}^{2+}$ release from mitochondria. J Neurosci 19: 3307-3315, 1999.

15. Hsu HC, Tang NY, Liu CH and Hsieh CL: Antiepileptic effect of Uncaria rhynchophylla and rhynchophylline involved in the initiation of c-Jun N-terminal kinase phosphorylation of MAPK signal pathways in acute seizures of kainic acid-treated rats. Evid Based Complement Alternat Med 2013: 961289, 2013.

16. Zhao P, Yang JM, Wang YS, Hao YJ, Li YX, Li N, Wang J, Niu Y, Sun T and Yu JQ: Neuroprotection of cytisine against cerebral ischemia-reperfusion injury in mice by regulating NR2B-ERK/CREB signal pathway. Neurochem Res 43: 1575-1586, 2018.

17. Liu X, Zhang X, Zhang J, Kang N, Zhang N, Wang H, Xue J, Yu J, Yang Y, Cui H, et al: Diosmin protects against cerebral ischemia/reperfusion injury through activating JAK2/STAT3 signal pathway in mice. Neuroscience 268: 318-327, 2014.

18. Zhang GX, Ge MY, Han ZW, Wang S, Yin J, Peng L, Xu F, Zhang Q, Dai Z, Xie L, et al: Wnt//-catenin signaling pathway contributes to isoflurane postconditioning against cerebral ischemia-reperfusion injury and is possibly related to the transforming growth factor $\beta 1 / \mathrm{Smad} 3$ signaling pathway. Biomed Pharmacother 110: 420-430, 2019.

19. Anastas JN and Moon RT: WNT signalling pathways as therapeutic targets in cancer. Nat Rev Cancer 13: 11-26, 2013.

20. Chen X, Wang CC, Song SM, Wei SY, Li JS, Zhao SL and Li B: The administration of erythropoietin attenuates kidney injury induced by ischemia/reperfusion with increased activation of Wnt/ß-catenin signaling. J Formos Med Assoc 114: 430-437, 2015.

21. Kuncewitch M, Yang WL, Molmenti E, Nicastro J, Coppa GF and Wang P: Wnt agonist attenuates liver injury and improves survival after hepaticischemia/reperfusion. Shock 39: 3-10, 2013.

22. He X, Mo Y, Geng W, Shi Y, Zhuang X, Han K, Dai Q, Jin S and Wang J: Role of Wnt/ $/$-catenin in the tolerance to focal cerebral ischemia induced by electroacupuncture pretreatment. Neurochem Int 97: 124-132, 2016.

23. Tremblay R, Hewitt K, Lesiuk H, Mealing G, Morley P and Durkin JP: Evidence that brain-derived neurotrophic factor neuroprotection is linked to its ability to reverse the NMDA-induced inactivation of protein kinase $\mathrm{C}$ in cortical neurons. J Neurochem 72: 102-111, 1999.

24. Bayne K: Revised guide for the care and use of laboratory animals available. American Physiological Society. Physiologist 39: 199208-199211, 1996.

25. Wu X, Li X, Liu Y, Yuan N, Li C, Kang Z, Zhang X, Xia Y, Hao Y and Tan Y: Hydrogen exerts neuroprotective effects on OGD/R damaged neurons in rat hippocampal by protecting mitochondrial function via regulating mitophagy mediated by PINK1/Parkin signaling pathway. Brain Res 1698: 89-98, 2018.

26. Zhang Y, Tao GJ, Hu L, Qu J, Han Y, Zhang G, Qian Y, Jiang CY and Liu WT: Lidocaine alleviates morphine tolerance via AMPK-SOCS3-dependent neuroinflammation suppression in the spinal cord. J Neuroinflammation 14: 211, 2017.

27. Livak KJ and Schmittgen TD: Analysis of relative gene expression data using real-time quantitative PCR and the 2(-Delta Delta C(T)) method. Methods 25: 402-408, 2011. 
28. Wang L, Zhang L and Chow BKC: Secretin prevents apoptosis in the developing cerebellum through Bcl-2 and Bcl-xL. J Mol Neurosci 68: 494-503, 2019.

29. Cai Y, Xu H, Yan J, Zhang L and Lu Y: Molecular targets and mechanism of action of dexmedetomidine in treatment of ischemia/reperfusion injury. Mol Med Rep 9: 1542-1550, 2014.

30. Zhang H, Cao HJ, Kimelberg HK and Zhou M: Volume regulated anion channel currents of rat hippocampal neurons and their contribution to oxygen-and-glucose deprivation induced neuronal death. PLoS One 6: e16803, 2011.

31. Khaspekov L, Friberg H, Halestrap A, Viktorov I and Wieloch T: Cyclosporin A and its nonimmunosuppressive analogue N-Me-Val-4-cyclosporin A mitigate glucose/oxygen deprivation-induced damage to rat cultured hippocampal neurons. Eur J Neurosci 11: 3194-3198, 1999.

32. Hou QL, Wang Y, Li YB, Hu XL and Wang SL: Protective effect of notoginsenoside R1 on neuron injury induced by OGD/R through ATF6/Akt signaling pathway. Zhongguo Zhong Yao Za Zhi 42: 1167-1174, 2017 (In Chinese).

33. Wei R, Zhang R, Li H, Li H, Zhang S, Xie Y, Shen L and Chen F: MiR-29 Targets PUMA to suppress oxygen and glucose deprivation/reperfusion (OGD/R)-induced cell death in hippocampal neurons. Curr Neurovasc Res 15: 47-54, 2018.

34. Tian X, An R, Luo Y, Li M, Xu L and Dong Z: Tamibarotene improves hippocampus injury induced by focal cerebral ischemia-reperfusion via modulating PI3K/Akt pathway in rats. J Stroke Cerebrovasc Dis 28: 1832-1840, 2019.

35. Zhang HY, Song YM and Feng C: Improvement of cerebral ischemia/reperfusion injury by daucosterol palmitate-induced neuronal apoptosis inhibition via PI3K/Akt/mTOR signaling pathway. Metab Brain Dis: May 4, 2020 (Epub ahead of print).
36. Zhang Y, Geng J, Hong Y, Jiao L, Li S, Sun R, Xie Y, Yan C, Aa J and Wang G: Orally administered crocin protects against cerebral ischemia/reperfusion injury through the metabolic transformation of crocetin by gut microbiota. Front Pharmacol 10: 440, 2019.

37. Seyfried FJ, Adachi N and Arai T: Suppression of energy requirement by lidocaine in the ischemic mouse brain. J Neurosurg Anesthesiol 17: 75-81, 2005.

38. Zheng X, Chen L, Du X, Cai J, Yu S, Wang H, Xu G and Luo Z: Effects of hyperbaric factors on lidocaine-induced apoptosis in spinal neurons and the role of $\mathrm{p} 38$ mitogen-activated protein kinase in rats with diabetic neuropathic pain. Exp Ther Med 13: 2855-2861, 2017.

39. Wu J, Yang J, Liu Q, Wu S, Ma H and Cai Y: Lanthanum induced primary neuronal apoptosis through mitochondrial dysfunction modulated by $\mathrm{Ca}^{2+}$ and Bcl-2 family. Biol Trace Elem Res 152: 125-134, 2013.

40. Ghosh AP, Walls KC, Klocke BJ, Toms R, Strasser A and Roth KA: The proapoptotic BH3-only, Bcl-2 family member, Puma is critical for acute ethanol-induced neuronal apoptosis. J Neuropathol Exp Neurol 68: 747-756, 2009.

41. Khodapasand E, Jafarzadeh N, Farrokhi F, Kamalidehghan B and Houshmand $\mathrm{M}$ : Is $\mathrm{Bax} / \mathrm{Bcl}-2$ ratio considered as a prognostic marker with age and tumor location in colorectal cancer? Iran Biomed J 19: 69-75, 2015

42. Jin Z, Ke J, Guo P, Wang Y and Wu H: Quercetin improves blood-brain barrier dysfunction in rats with cerebral ischemia reperfusion via Wnt signaling pathway. Am J Transl Res 11: 4683-4695, 2019.

c) (i) $\odot$ This work is licensed under a Creative Commons

c) EY NC ND Attribution-NonCommercial-NoDerivatives 4.0 International (CC BY-NC-ND 4.0) License. 